\title{
INVERSION OF THE UTERUS DURING METHOXYFLURANE ANAESTHESIA
}

\author{
H. BalaKrishna, M.D., and Gertie F. Marx, M.D."
}

ACUTE INVERSION OF THE UTERUS is a rare but important cause of postpartum shock. ${ }^{1}$ Three prerequisites are necessary for its development: (1) atony or thinness of the uterine wall, (2) sudden emptying of the uterine cavity, and (3) a dilated cervix.1,2 Inversion may occur spontaneously as a result of an abrupt contraction of the abdominal muscles or pressure from the weight of the intestines, or may be caused by traction on the cord or membranes or an over-vigorous Credé manoeuvre. ${ }^{1-3}$ In a study of 166 puerperal patients with acute inversion, 83 cases were spontaneous, 44 followed traction on the cord, and 39 were attributable to improper expression of the placenta. ${ }^{4}$ This report concerns a case of postpartum uterine inversion in which deep methoxyflurane (Penthrane ${ }^{\circledR}$ ) anaesthesia may have been a contributing factor.

\section{Case Report}

A 23-year-old healthy primigravida was brought to the delivery room with her cervix fully dilated. Her prenatal course had been uncomplicated. Pain relief during labour was provided with two intramuscular doses of meperidine (Demerol $\left.{ }^{\circledR}\right) 25 \mathrm{mg}$. and one dose of promethazine (Phenergan ${ }^{\circledR}$ ) $25 \mathrm{mg}$. The patient was induced and maintained with methoxyflurane-oxygen in a semi-closed system using a Pentec vaporizer. An oropharyngeal airway was inserted and respirations were assisted. The plane of anaesthesia was assessed as "moderately deep." After 20 minutes of anaesthesia, a 3360 gm. infant was delivered by low-mid forceps over a medio-lateral episiotomy; one-minute Apgar score was 8, five-minute score 10. The placenta delivered spontaneously six minutes after the birth of the infant and was complete. However, the patient was bleeding, and pitocin $20 \mathrm{mg}$., dissolved in $500 \mathrm{ml}$. of 5 per cent dextrose in water, was infused rapidly. To examine for a laceration, it was necessary to rotate the cervix forward and, simultaneously, move the fundus out of extreme anteflexion by a light pressure on the abdomen. A $1 \mathrm{~cm}$. Iaceration was found. The vaginal mucosa of the episiotomy was repaired first. When this was completed, and the cervical tear was to be sutured, the cervix was no longer palpable in the vagina. Instead, a hard mass, about $8 \mathrm{~cm}$. in diameter, was felt. Concurrently, the patient became cold and clammy, her blood pressure fell from 110/60 to 60/?, and the pulse rate rose from 88 to 132 . At this time, anaesthesia had been administered for 46 minutes. A diagnosis of uterine inversion was made. The uterus soon became boggy and began to bleed heavily. Trendelenburg position (15 ) was instituted; anaesthesia was discontinued and "wash-out" of the methoxyflurane

${ }^{\circ}$ Department of Anesthesiology, Albert Einstein College of Medicine, Bronx, New York. 
attempted. Dextran $500 \mathrm{ml}$. was infused rapidly followed by $500 \mathrm{ml}$. of whole blood. Within 20 minutes, the blood pressure increased to $100 / 60$ and the pulse rate declined to 100 . The patient, who was moving by now, was reanaesthetized with the same technique, and the inversion was slowly corrected. Oxytocin was injected intravenously, and the uterus massaged lightly until it contracted. The uterine cavity was then explored, but no rupture or abnormalities were noted. Repair of the episiotomy was completed. The patient left the delivery room with a blood pressure of $110 / 60$, pulse 92 . Recovery was uneventful.

\section{Discussion}

Methoxyflurane offers substantial advantages in obstetric anaesthesia. It has potent analgesic properties, is associated with a low incidence of laryngospasm and vomiting, and is non-explosive. In a large number of unselected vaginal deliveries in which the agent was used to potentiate nitrous-oxide-oxygen, no untoward effects were reported in either the mother or the neonate. ${ }^{5-7}$ Uterine contractility, as determined by clinical evaluation or external tokography, was not altered when "analgesic" concentrations were inhaled.,8 However, when the concentration was high or the administration prolonged, a gradual decrease in the amplitude of contractions with no change in the frequency was noted by intrauterine pressure recording. ${ }^{9}$

In a recent report involving uterine inversion following a breech delivery, the authors concluded that halothane (Fluothane ${ }^{(1)}$ ), by virtue of its potential for uterine and cervical relaxation, could set the stage for uterine inversion. ${ }^{10}$ They advocated caution in managing the third stage of labour whenever halothane anaesthesia is used. The same recommendation appears indicated when methoxyflurane has been administered in anaesthetic concentrations and/or for prolonged periods.

Inversion of the uterus is followed by vasomotor collapse. Usually, shock is out of proportion to the amount of blood lost, although profuse haemorrhage may occur. Treatment consists of manual replacement of the uterus which should be performed as early as possible. ${ }^{1,2}$ However, if blood loss has been severe, manipulation should be postponed until after transfusion. Since uterine reposition is painful, anaesthesia should be employed and must be deep as there is a tendency to spasm of the constricting supracervical portion. ${ }^{2}$ Careful uterine exploration should be undertaken after the manual procedure to ascertain that perforation has not occurred. ${ }^{10}$

\section{SUMMARY}

A case of postpartum uterine inversion is presented as a possible complication of prolonged methoxyflurane-oxygen anaesthesia.

\section{REFERENCES}

1. Eastman, N. J. \& Heliman, L. M. Williams Obstetrics, 12th ed., New York: AppletonCentury-Crofts (1961), chapter 35: Abnormalities of the Third Stage of Labor, pp. 995-1010. 
2. Greenhill, J. P. Obstetrics. 12th ed., Philadelphia: Saunders (1961), chapter 65: Pathology of the Third Stage, pp. 793-825.

3. Bunke, J. W. \& Hofmeister, F. J. Uterine Inversion: Obstetrical Entity or Oddity. Am. J. Obst. \& Gynec. 91: 934 (1965).

4. DAs, P. Inversion of Uterus. J. Obst. \& Gynaec. Brit. Emp. 47: 525 (1940).

5. Romagnoli, A. \& Korman, D. Methoxyflurane in Obstetrical Anaesthesia and Analgesia. Canad. Anaesth. Soc. J. 9: 414 (1962).

6. BoIsvert, M. \& Hudon, F. Clinical Evaluation of Methoxyflurane in Obstetrical Anaesthesia: A Report on 500 Cases. Canad. Anaesth. Soc. J. 9: 325 (1962).

7. Schubert, F. \& Keuter, J. Experience with Methoxyflurane in Obstetrical Anesthesia. Proc. Third World Congr. Anesth. 2: 25 (1964).

8. Miller, J. R. \& Stoelting, V. K. In Vivo and In Vitro Studies of Penthrane for Obstetrical Deliveries. Presented at the Work Completed Session, Annual Meeting of the American Society of Anesthesiologists, Philadelphia (1966).

9. WinNER, W. \& MARX, G. F. Unpublished data.

10. Harrus, R. E. \& DunNiroo, D. R. Inversion of the Uterus in a Patient under Halothane Anesthesia. Obst. \& Gynec. 27: 655 (1966). 